\title{
DEVELOPMENT OF A SUPERCONDUCTING CONNECTION FOR NIOBIUM CAVITIES*
}

\author{
P. Kneisel ${ }^{\#}$, G. Ciovati, J. Sekutowicz, TJNAF, Newport News, Virginia, USA \\ A. Matheisen, X. Singer and W. Singer, DESY,Hamburg, Germany
}

\begin{abstract}
Several, partially successful attempts have been made in the past to develop a superconducting connection between adjacent niobium cavities with the capability to carry up to $30 \mathrm{mT}$ of the magnetic flux. Such a connection would be particularly of great benefit to layouts of long accelerators like ILC because it would shorten the distances between structures and therefore the total length of an accelerator with the associated cost reductions. In addition the superconducting connection would be ideal for a super-structure - two multi-cell cavities connected through a half wavelength long beam pipe providing the coupling. Two welded prototypes of super-structure have been successfully tested with beam at DESY. The chemical treatment and water rinsing was rather complicated for these prototypes because of the length of the assembly. We have engaged in a program to develop such a connection, initially based on the Nb55Ti material. Several options are pursued e.g. a two-cell cavity is being used to explore the reachable magnetic flux for the TESLA like connection with a squeezed niobium gasket between the flanges. Other materials, such as $\mathrm{NbZr}$ or $\mathrm{NbN}$ are also being considered. In this contribution, we will report about the progress of our investigations.
\end{abstract}

\section{INTRODUCTION}

A superconducting connection between adjacent cavities in a cavity string has been desired for a long time and several attempts have been made in the past to develop such a system. The reasons are as obvious as the technological difficulties: a reliable superconducting connection would allow a shortening of the distances between adjacent cavities - presently connections are being made at locations, where the cavity fields decay to a level, which generates only minute losses - providing a more compact design with the associated cost reductions. In addition, it would be possible to manufacture the accelerating cavities - typically consisting of a rotationally symmetric center portion (the cells) and asymmetric end groups - more cost effective by e.g. applying "seamless" fabrication technologies [1] for the rotational symmetric center portion (Fig. 1).

However, these connections have to be able to carry "loss-free" currents corresponding to magnetic field levels

\footnotetext{
* This manuscript has been authored by Jefferson Science Associates, LLC under U.S. DOE Contract No. DE-AC05-06OR23177. The U.S. Government retains a non-exclusive, paid-up, irrevocable, world-wide license to publish or reproduce this manuscript for U.S. Government purposes.

${ }^{\#}$ kneisel@jlab.org
}

up to $30-40 \mathrm{mT}$ in the case of e.g. a superstructure as shown in Figure 2. Because of the surface oxides, the topology of the flanges and gaskets and the mechanical properties of the materials in such a connection, Josephson junction like systems will develop, which can or cannot sustain the required fields.

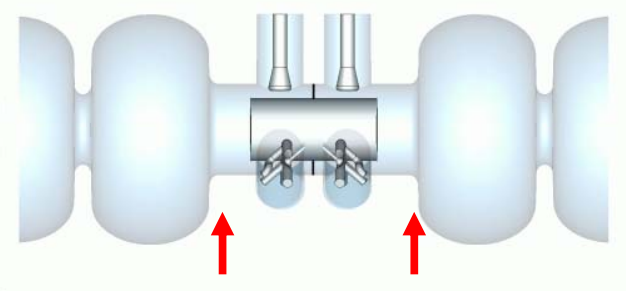

Superconducting Joints

Figure 1: Flanged end-groups.

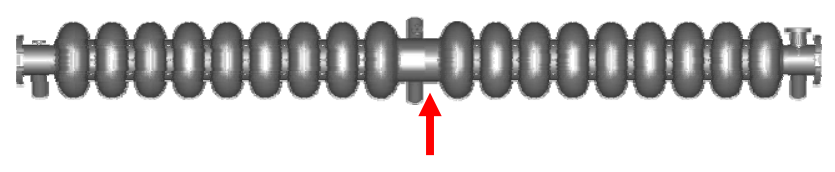

Superconducting Joint

Figure 2: Super-Structure configuration [2].

In addition, the development of superconducting cavities has gone in the direction of immersing only the "cell section" of a cavity in liquid helium, whereas the end groups (fundamental power coupler, higher order mode couplers) are cooled by conduction only. A superconducting joint would therefore be placed outside the helium bath and therefore a high thermal conductivity of the joint materials is essential.

A serious effort in developing a superconducting connection between adjacent cavities was made 30 years ago at the Kernforschungszentrum Karlsruhe for the Karlsruhe/CERN particle separator [3]. A separator section of $2.4 \mathrm{~m}$ length was designed to consist of 5 app. $60 \mathrm{~cm}$ long subunits, which were connected together in a "joint cell", which had somewhat lower fields than the "deflecting" cells. Nevertheless, this connection had to carry some currents. A special "joint ring with lips", made from niobium, was developed, which, when pressed between the flanges of the subunits, produced some shearing movement between the lips, tearing the natural oxide layer on the niobium and produced some good metallic contacts. The functions of RF contact and providing a leak tight connection were separated by the 
use of indium wires for the vacuum integrity. Surface roughness of both the flanges and "joint ring" were important and metallographic studies showed point-like contacts between the flanges and the joint ring. Even though this approach satisfied the requirements for the separator project, this connection could safely only be used to field levels of 3-5 mT.

Another approach in the past for connecting a cavity string has been to make the joint-cell "field-free" (very low fields), i.e. the RF-contact is not exposed to currents and fields. In this case a lower quality RF joint is possible, which has been applied by means of an indium wire at the Superconducting Accelerator (SCA) at Stanford University [4]. Indium seems to be a good choice for contacts because of its easy handling, however, the critical fields are low - app. $15 \mathrm{mT}$ at $2 \mathrm{~K} \mathrm{[5]}$ and therefore not useful for the superstructure approach.

For this application, a series of tests has been conducted at DESY on a two cell cavity, on which the superconducting joint was placed very close to the iris of one end-cell, being exposed to $\sim 20 \%$ of the fields in the cell on axis. The flange configuration was a copy of the "standard" NbTi - flange on the TESLA cavities [6] with the replacement of the AlMg - "diamond" shaped gasket by a niobium gasket. In some of the tests magnetic fields up to $\sim 10 \mathrm{mT}$ could be sustained, however, the reproducibility was quite poor. Eventually the superstructure [2] consisting of two 7-cell cavities, was electron beam welded together instead of flanged with a sc joint.

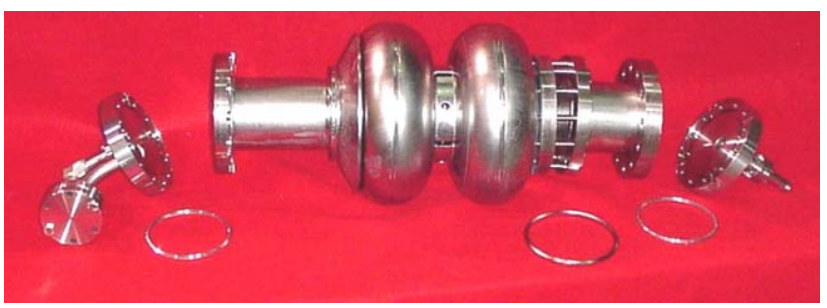

Figure 3: Two-cell cavity with superconducting joint.

For the ANL ATLAS accelerator some work was carried out to develop a demountable superconducting joint, based on indium and niobium as gasket material [7].

In both cases the development efforts did not result in a useable configuration above $\sim 2-3 \mathrm{mT}$; however, it became clear that a good sc joint needed to penetrate the natural oxide layers on the flange and gasket materials.

\section{PRESENT APPROACH}

\section{Split $T M_{010}$ Cavity}

We started out optimistically with our development by fabricating a $\mathrm{TM}_{010}$ cavity at $2.3 \mathrm{GHz}$ as shown in figure 4, which was split in the middle and to each half cell a conflat flange made from Nb55Ti was welded on. Gaskets were made of niobium. This cavity type had been used successfully for the development of the Karlsruhe separator joint with the exception that the flat flanges on the half-cells were made from niobium. In this configuration, the maximum current is flowing across the joint and the idea was that the knife edges from the conflat would "dig" sufficiently into the niobium oxide on the gasket to provide good contact. Even though the Conflat flanges and the niobium gasket worked very well for vacuum purposes, we were not able to establish a reasonable field in the cavity because multipacting (MP) occurred at very low power levels, which could not be processed away. MP simulation calculation did not find any barriers anywhere in the cavity. Of course, the suspicion was MP between the flanges and the gasket because of a slight gap in the design.

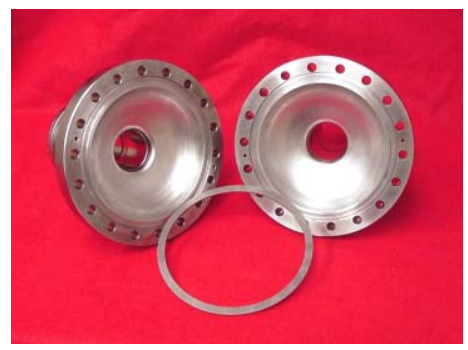

Figure 4: $\mathrm{TM}_{010}$ split cavity.

\section{Two-Cell DESY Cavity}

The 2-cell cavity of the TESLA shape (Fig. 3), which had been used at DESY for sc joint investigations as mentioned previously, was also used in our initial investigations with the modification that the diamond shaped gasket was made from single crystal niobium. We hoped that the elimination of grain boundaries on the gasket would improve the current carrying capability of the joint. However, these tests were not successful either and our attention/suspicion was directed towards the choice of the flange material. Is the Nb55Ti capable of withstanding magnetic field levels of the order of $\sim 30 \mathrm{mT}$ as needed for e.g. the superstructure?

\section{Single Cell Cavities from Different Material}

In order to explore the capabilities of the potential flange materials we fabricated a $1.5 \mathrm{GHz}$ single cell of the CEBAF Upgrade High-Gradient shape [8] from Nb55Ti. In addition, we attempted to do the same with a $1.3 \mathrm{GHz}$ TESLA-shape single cell from $\mathrm{Nb} 1 \% \mathrm{Zr}$ [9], a material, which is readily available from W.C. Heraeus and which had been considered at DESY as a possible alternative flange material to Nb55Ti. We encountered manufacturing problems during the deep drawing of the half cells and could only successfully form one half cell for the single cell cavity. We completed the cavity by making the other half from large grain niobium.

The test with these cavities were "eye-opening": as is shown in Fig. 5, the NbTi cavity could - after typical BCP used for niobium - only support a magnetic field of $\sim 7$ $\mathrm{mT}$, whereas the $\mathrm{NbZr}$ - cavity quenched at $42 \mathrm{mT}$, a sufficiently high enough magnetic field for a superstructure configuration (Fig. 5). We suspect that the very low field in the NbTi cavity was possibly caused by segregation of the Ti rich $\alpha$-phase in the electron beam 
weld with associated Ti rich clusters. Unfortunately our on-going investigations of the composition in weld area could not prove this yet.

A literature search also revealed that the thermal conductivity of $\mathrm{NbTi}$ is very poor $[10]$ - about a factor of 200 smaller than for pure niobium. The residual resistance, obtained from a fit of the $\mathrm{R}_{\mathrm{s}}(\mathrm{T})$ data, was also about a factor of 20 higher than typically observed for pure niobium. Calculations using the thermal feedback model [11] estimated a quench field of $8 \mathrm{mT}$, in good agreement with the data.

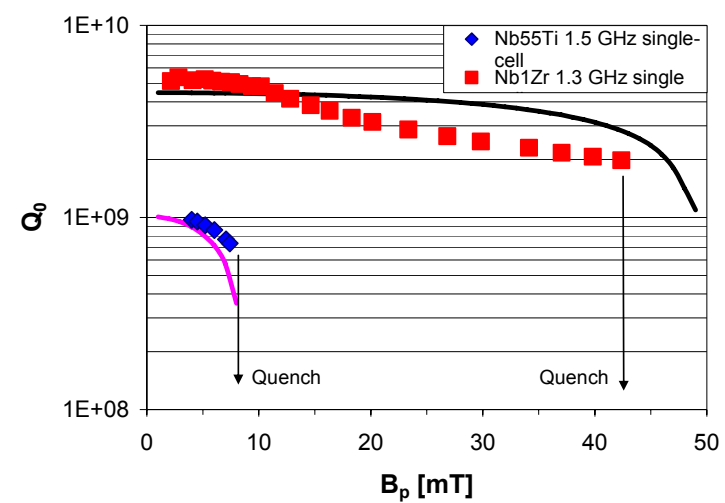

Figure 5: Quench fields measured in Nb55Ti and $\mathrm{Nb} 1 \% \mathrm{Zr}$ cavities at $2 \mathrm{~K}$. Solid lines show the results from calculations with the thermal feedback model.

Another possible flange material with very good thermal and SRF properties would be $\mathrm{NbN}$ in the form of a thin layer backed by $\mathrm{Nb}$. Nitridation of material for hardening is routinely done for e.g. tooling; nitrified $\mathrm{Nb}$ also shows increased hardness and would be a good material for knife edge flanges.

We have done an initial hardness test on nitrified $\mathrm{Nb}$, accomplished by exposing niobium at $\sim 1200{ }^{\circ} \mathrm{C}$ to a nitrogen atmosphere for several hours. The hardness increased to 150 on the Vickers scale.

From experiments many years ago we still had a nitrified niobium single cell cavity in storage, which we retested after high pressure rinsing only and clean room assembly. The very encouraging result is shown in Fig. 6: a high Q-value at $2 \mathrm{~K}$ was measured with little slope and the cavity could sustain magnetic peak fields up to $65 \mathrm{mT}$.

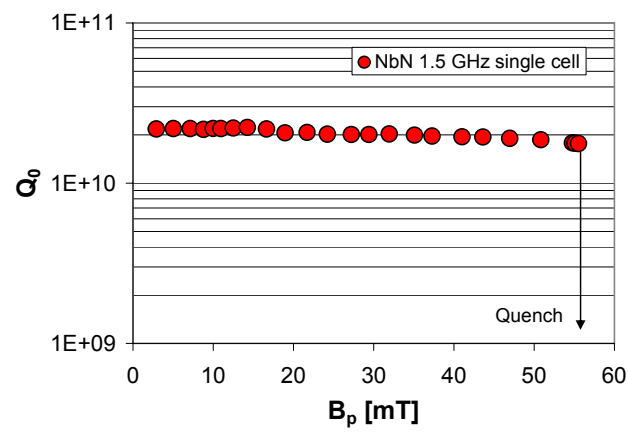

Figure 6: Performance of $\mathrm{NbN}$ cavity measured at $2 \mathrm{~K}$.

\section{FUTURE PLANS}

Our future work foresees the implementation of $\mathrm{NbZr}$ knife-edge flanges on both the split cavity and the 2-cell DESY cavity. In addition we are designing a test with two single cell cavities, which will be connected to each other through a sc joint consisting of NbZr flanges and a niobium gasket, fully immersed in liquid helium. In a second configuration, we will shield the flanges/gasket from the helium bath - the "real life" situation in a cryomodule - and determine the performance of the joint, if only conduction cooling is available.

In parallel, we will follow the promising route of developing processing parameters for $\mathrm{NbN}$ layers on niobium by either thermal nitrification or plasma nitrification with emphasis on obtaining good superconducting properties. As a first step, we will nitrify conflat flanges made from niobium under different conditions and measured the hardness and the deformation after several assemblies of the flanges. Nitridation of cavities will follow.

\section{ACKNOWLEDGEMENT}

We would like to thank our colleagues from Jefferson Lab - L. Turlington, R. Manus, G. Slack, S. Manning,T. Elliott and P. Kushnick - for their support of this work.

\section{REFERENCES}

[1] W.Singer, Physica C 441 (2006) 89.

[2] J. Sekutowicz, M. Ferrario and Ch. Tang, Phys. Rev. ST AB 2 (1999) 062001.

[3] M. Grundner et al., Nucl.Instr. \& Meth. 141 (1977) 57.

[4] M.McAshan et al.; HEPL Report 665, Stanford (1972).

[5] Y.Bruynseraede et al., Physica 54 (1971) 137.

[6] K. Zapfe-Dueren et al.; "A New Flange Design for the Superconducting cavities for TESLA", Proc. $8^{\text {th }}$ SRF workshop, Abano Terme, Italy (1997), Report LNL-INFN 133/98, p.457.

[7] K. Shephard et al.; "ATLAS Design Notes: Superconducting RF Demountable Joint" (2005).

[8] J. Sekutowicz et al., "Cavities for JLAB's $12 \mathrm{GeV}$ Upgrade", in Proc. of the 2003 Particle Accelerator Conference, Portland OG, USA, 1395 (2003).

[9] H. Kaiser et al.; "Materials for the Flanges of the TESLA Cavity", TESLA Report 99-04.

[10] E. W. Collings, “Applied superconductivity, metallurgy and Physics of Titanium Alloys", Plenum Press, New York, 1986.

[11] A. Gurevich, Physica C 441 (2006) 38. 